\title{
Anabases
}

ANABASES Traditions et réceptions de l'Antiquité

$20 \mid 2014$

Varia

\section{Entre philologie, anthropologie et histoire des religions. Entretien avec Claude Calame}

\section{(2) OpenEdition \\ Journals}

Édition électronique

URL : http://journals.openedition.org/anabases/5112

DOI : 10.4000/anabases.5112

ISSN : 2256-9421

Éditeur

E.R.A.S.M.E.

\section{Édition imprimée}

Date de publication : 1 novembre 2014

Pagination : 345-375

ISSN : $1774-4296$

Référence électronique

"Entre philologie, anthropologie et histoire des religions. Entretien avec Claude Calame », Anabases [En ligne], 20 | 2014, mis en ligne le 01 novembre 2017, consulté le 14 novembre 2019. URL : http:// journals.openedition.org/anabases/5112; DOI : 10.4000/anabases.5112 


\section{Entre philologie, anthropologie et histoire des religions}

Entretien avec Claude Calame

\section{Note introductive}

LE MARDI 12 NOVEMBRE 2013, l'équipe PLH-ERASME accueillait Claude Calame pour un échange consacré à son long parcours dans les domaines de la philologie grecque, de l'histoire ancienne et de l'anthropologie comparée ${ }^{1}$. L'ensemble de ses recherches est aussi marqué par un profond intérêt pour tout ce qui concerne la réception de l'Antiquité. Claude Calame est un chercheur soucieux de reconstituer les liens entre Anciens et Modernes, entre le passé de l'Antiquité et le présent des usages qui en sont issus. C'est à tous ces titres qu'il s'est volontiers prêté au jeu de l'échange et du débat.

Rappelons en quelques dates ce qu'a été le cheminement de l'enseignant et du chercheur. Claude Calame a mené à bien des recherches doctorales, dans le cadre d'une thèse d'État, sur une institution culturelle : les chours de jeunes filles en Grèce archaïque, et sur la mise au point d'une édition critique de l'ouvre du poète Alcman. Ces travaux sont l'objet de deux volumes qui paraissent en 1977. Il est ensuite professeur de lycée, de 1975 à 1984. Puis, pendant presque vingt ans, de 1984 à 2003, il occupe la chaire de professeur de langue et littérature grecques à l'université de Lausanne. À partir de 2001 et jusqu'en 2013, il est directeur d'Études à l'École des Hautes Études en Sciences Sociales (EHESS, Paris), et désormais directeur d'Études émérite. Au cours de toutes ces années, Claude Calame déploie une très intense activité de recherche et d'enseignement, en Suisse et dans de nombreux pays, qui se traduit par une impressionnante série d'articles, d'écrits, de livres, de prises de position aussi.

1 La transcription, avec l'appareil de notes, a été réalisée par Marylène Lebrère et revue par Anthony Andurand, Corinne Bonnet et Pascal Payen. L'ensemble a fait l'objet d'une révision par Claude Calame. 
Pascal Payen : Pour prendre une vue d'ensemble de vos recherches et de vos activités, je soulignerai tout d'abord que la constante la plus remarquable de vos travaux, depuis les années 1970, est l'entrecroisement entre science et engagement. Or, lorsque l'on travaille sur un domaine en apparence aussi déconnecté de nos réalités quotidiennes que l'Antiquité grecque, cette association ne va pas de soi. La science que vous maîtrisez en priorité est la philologie classique la plus rigoureuse, la plus austère, celle dont vous n'hésitez pas à dire vous-même qu'elle procure parfois ennui, ou qu'il lui arrive d'être peu productive. L'engagement, lui, est de nature politique, au sens grec. Il concerne la place du savant dans la cité et il tient notamment en quelques idées fortes, vraies pour la Grèce ancienne et pour le temps présent, sur la fonction éducatrice de la culture qui garantit et renforce la cohésion du groupe et qui inspire ses valeurs. Ces convictions se prolongent avec le regard porté sur les évolutions récentes des sociétés contemporaines, en particulier ce que vous nommez la " culture de supermarché ${ }^{2}$ " envahissante. Les premiers travaux de grande ampleur, la thèse sur les chœurs de jeunes filles, puis l'édition d'Alcman, entrent en résonance, dans la méthode même, avec ces convictions. Par exemple, dans ce passage de l'introduction à l'édition d'Alcman. Je le cite :

" [...] au-delà de toute théorie du reflet, la production d'un auteur ne peut être comprise sans la prise en compte du cadre social et culturel constituant le contexte de son énonciation et de sa transmission ${ }^{3}$ ".

Le contexte est ici celui de la Sparte de la fin VII ${ }^{\mathrm{e}}$ siècle avant J.-C., où s'est développée l'activité d'Alcman. La culture n'est donc pas une entité en soi, ou d'ordre supérieur. En Grèce, par exemple, musique et poésie, écrivez-vous, ne sont pas développées comme des activités de pur divertissement, et l'exécution musicale est toujours attachée à une occasion publique. Le passage qui suit explicite avec lucidité que l'activité culturelle est indissociable de son implantation sociale et il montre en quoi la position du poète grec, ici Alcman, annonce le rapport qu'entretient le savant moderne entre science et engagement. Je vous cite encore, il s'agit d'un passage un peu long, mais qui mérite que l'on en prenne une connaissance d'ensemble :

"Il y a donc non seulement une correspondance probable entre les deux "institutions" dont la musique spartiate a été l'objet au cours du VII ${ }^{\mathrm{e}}$ siècle et la restructuration des festivals civiques à la même époque [les Gymnopédies et les Carnéia] mais aussi un lien étroit entre le caractère politique et religieux de la production poétique spartiate de cette époque et la valeur politique des festivals au cours desquels ces poèmes étaient chantés. Le

2 C Calame, " Les sciences de l'Antiquité entre néolibéralisme et culture de supermarché : inflation bibliographique et égarement méthodologique ", in Sentiers transversaux. Entre poétiques grecques et politiques contemporaines, Grenoble, Jérôme Millon, 2008, p. 219-236.

3 C. Calame, Alcman. Texte critique, témoignages, traduction et commentaire, Rome, Ateneo, 1984, p. XI. 
poète spartiate joue un rôle précis dans la société qui l'emploie et qui, souvent, le fait venir de l'étranger ; dans une culture reposant encore essentiellement sur une tradition orale, il assure la transmission de l'idéologie destinée à perpétuer et à maintenir les structures morales de la communautét."

C'est la raison pour laquelle la seconde caractéristique majeure de vos travaux réside dans le fait qu'ils mobilisent un vaste ensemble de disciplines dans le champ des sciences humaines et sociales. Et l'on peut déplorer avec vous que certains savants et hellénistes s'en étonnent encore. Par cet usage, il n'est pas question de "faire moderne », Internet aidant. Le but visé est de redonner au contexte historique et énonciatif sa diversité et sa complexité, en particulier les voies discursives qui ont laissé leurs traces dans les textes de la Grèce ancienne. C'est lui donner, à ce contexte, une fonction à part entière dans le processus de création artistique et dans le fonctionnement des institutions politiques des pratiques culturelles et des comportements sociaux. Un des exemples les plus probants de cette démarche se trouve certainement dans l'ample analyse de la figure du héros athénien Thésée, analyse réunie et déployée dans un livre singulier, Thésée et l'imaginaire athénien. Légende et culte en Grèce antique, paru en $1990^{5}$. On peut ne pas être d'accord au total avec ce livre, mais que l'on prenne, auparavant, le temps de le lire dans le détail et que l'on prenne le temps d'argumenter.

Dans ce livre, les mythes et les rites qui se rattachent à Thésée, à partir de la fin $\mathrm{du} \mathrm{VI}^{\mathrm{e}}$ siècle et au $\mathrm{V}^{\mathrm{e}}$ avant J.-C., sont l'objet d'une profonde reformulation et réorganisation ; ils s'inscrivent dans le contexte du développement de l'Athènes démocratique, de la définition et de l'extension de son espace égéen, de la fabrication de son panthéon autour des figures de Poséidon, d'Athéna et d'Apollon. Ils sont donc l'objet d'une réactivation des rapports entre mythes et rites qui sont au cœur de l'analyse. Le livre montre que toute manifestation sociale, toute pratique culturelle, tout processus historique ne peuvent être appréhendés qu'en prenant en compte les circonstances de leur élaboration. Au point que dans le premier chapitre du livre, la notion de "circonstant " devient un des actants de l'ensemble du processus symbolique. Les trois niveaux autour desquels s'élabore ce processus historique sont les suivants : tout d'abord, les formes de culture s'enracinent dans un cadre institutionnel et plus largement dans une occasion déterminée qui doit faire l'objet d'une analyse historique attentive à la spécificité de chaque situation. On peut alors parler d'une histoire contrastive. Au deuxième niveau, ces expériences sont ensuite rapportées, véhiculées en des langages, avec leurs codes culturels propres, qu'on les appelle lyriques chorales, tragédies, proses historiographiques, iconographie, décrets de l'ekklèsia, qui appartiennent tous à des formes de discours répertoriées par la poétique des genres et par la philologie. Enfin, un troisième niveau : ces manifestations historiques, sociales et discursives sont l'objet

4 Ibid., p. XIII-XIV.

5 C. Calame, Thésée et l'imaginaire athénien. Légende et culte en Grèce antique, Lausanne, Payot, 1990 (2éd. : 1996). 
d'un processus de transmission. Par là, elles sont comme prises en charge par la société, elles sont reçues tout au long des différentes étapes de la tradition, marquées par un processus de réappropriation dans une perspective d'efficacité pragmatique. Ainsi, on voit se définir les différents composants de ce que j'appellerais volontiers une anthropologie successivement historique, poétique, pragmatique.

Je ne sais si Claude Calame se retrouvera dans mon propos, à coup sûr trop rapide, trop simplificateur; du moins est-ce ainsi que je vous ai lu depuis la recension de votre Thésée que m'avait demandée Nicole Loraux. Et je suis heureux de vous associer dans ce qui reste, pour moi, une expérience intellectuelle marquante ${ }^{6}$.

Quelles sont les principales étapes, sous la forme de livres, qui ont jalonné vos recherches ? Je voudrais rappeler quelques-unes de ces étapes, pour les porter à la connaissance de tous, des étudiants en particulier, non pas dans un ordre chronologique, mais plutôt selon un parcours thématique, parmi d'autres possibles. Tout d'abord, les choix méthodologiques que je viens de relever autour du problème de la place du contexte historique et social, perceptible dans toute œuvre de culture, sont déjà présents dans votre recherche doctorale publiée sous le titre Les Chours de jeunes filles en Grèce archaïque. I: Morphologie, fonction religieuse et sociale et II : Alcman. Ces recherches sont prolongées dans l'édition d'Alcman qui paraît quelques années plus tard en 1984. Ce problème de l'efficacité pragmatique de la fiction et des différents masques d'autorité qu'elle véhicule et dissimule est repris dans l'étude de corpus plus larges et plus diversifiés allant de la poésie mélique à la tragédie, des poèmes hésiodiques à la prose d'Hérodote, dans trois livres, au moins, si je m'en tiens uniquement à ceux qui ont été écrits par vous d'une seule main. C'est d'abord Le récit en Grèce ancienne ${ }^{7}$; il s'agit ensuite d'un livre paru en 2005 intitulé Masques d'autorité. Fiction et pragmatique dans la poétique grecque antique ${ }^{8}$. Il s'agit enfin d'un livre intitulé Poétique des mythes en Grèce antique, paru en 2000, où sont appliqués aux mythes grecs des outils théoriques de manière à mettre au jour la fonction de ces récits mythiques, leur destination sociale dans des occasions et des circonstances particulières ${ }^{9}$. Ce dernier livre étant plutôt destiné à un public d'étudiants.

6 P. PAYEN, "C. Calame, Thésée et l'imaginaire athénien. Légende et culte en Grèce antique (compte rendu) ", Revue de l'Histoire des Religions 210, 1 (1993), p. 93-99.

7 C. Calame, Le récit en Grèce ancienne. Énonciations et représentations de poètes, Paris, Klincksieck, 1986 ( $2^{\mathrm{e}}$ éd. revue et augmentée : Paris, Belin, 2000) ; trad. it. Il racconto in Grecia. Enunciazioni e Rappresentazioni di poeti, Rome-Bari, Laterza, 1988 ; éd. revue et trad. angl. The Craft of Poetic Speech in Ancient Greece, Ithaca NY - Londres, Cornell University Press, 1995.

8 C. Calame, Masques d'autorité. Fiction et pragmatique dans la poétique grecque antique, Paris, Les Belles Lettres, 2005 ; trad. angl. Masks of Authority. Fiction and Pragmatics in Ancient Greek Poetics, Ithaca NY - Londres, Cornell University Press, 2005.

9 C. Calame, Poétique des mythes en Grèce antique, Paris, Hachette, 2000 ; trad. angl. Greek Mythology : Poetics, Pragmatics and Fiction, Cambridge, Cambridge University Press, 2009 ; trad. it. : Poetiche dei miti nella Grecia antica, Lecce, Argo, 2011. 
Un troisième ensemble de livres se donne pour tâche d'étudier quelques grandes catégories qui ont organisé la vie et la pensée des Grecs. Ce sont les représentations qu'ils se sont forgées de l'espace et du temps dans un livre intitulé Pratiques poétiques de la mémoire. Représentations de l'espace-temps en Grèce ancienne et paru en $2006^{10}$. Ce sont ensuite les rapports entre mythe et histoire, à propos des récits de la fondation de Cyrène dans Mythe et histoire dans l'Antiquité grecque, livre publié d'abord en 1996 et qui a fait l'objet d'une refonte récente ${ }^{11}$. Celles et ceux qui voudraient avoir une vue d'ensemble de vos travaux pourront lire le recueil de quinze articles publié en 2008, Sentiers transversaux. Entre poétiques grecques et politiques contemporaines ${ }^{12}$.

Partant de ces quelques remarques, j'ouvrirai nos discussions de cet après-midi en vous posant deux questions : tout d'abord, vos travaux, on l'a vu, s'échelonnent sur plusieurs décennies d'une activité accompagnée d'un constant recul critique concernant les catégories et les contextes auxquels vous recourez, à partir de cette position singulière que j’appellerai de " décalage engagé ». Quelle Grèce pensez-vous avoir forgée au long de vos recherches ? Ce n'est pas celle du " miracle grec » de Renan, ce n'est pas celle de la philologie classique, ce n'est pas non plus celle de l'anthropologie structurale pourvoyeuse de paradigmes englobants, touchant le politique et le religieux... Dès lors laquelle?

Deuxième question : comment pensez-vous vous situer parmi les traditions d'études sur la Grèce ancienne ? La voie de l'anthropologie historique pourrait-elle représenter ce dont vous vous sentez le plus proche? Quelles interférences la voie de l'anthropologie entretient-elle dans vos travaux avec celles de la philologie et de l'histoire?

Claude Calame : Tout d'abord, juste quelques mots pour remercier Pascal d'une présentation qui me touche beaucoup et qui offre des enjeux des travaux que j'ai pu " commettre " une présentation synthétique et pénétrante, dans des termes que moimême je n'aurais pas pu employer. Je suis vraiment très reconnaissant pour la présentation qu'il me tend en miroir.

Il me revient de répondre à ces grandes questions, d'abord sur la Grèce antique qu'il me semble avoir abordée, forgée, reconstruite, tout en étant sensible à l'aspect

10 C. Calame, Pratiques poétiques de la mémoire. Représentations de l'espace-temps en Grèce ancienne, Paris, La Découverte, 2006 ; trad. angl. Poetic and Performative Memory in Ancient Greece : Heroic Reference and Ritual Gestures in Time and Space, Cambridge Mass. - Londres, Harvard University Press, 2009.

11 C. Calame, Mythe et histoire dans l'Antiquité grecque. La création symbolique d'une colonie, Paris, Les Belles Lettres, $2011^{2}$ (1 $1^{\text {re }}$ éd. : Lausanne, Payot, 1996); trad. angl. : Myth and History in Ancient Greece, Princeton, Princeton University Press, 2003.

12 C. Calame, Sentiers transversaux. Entre poétiques grecques et politiques contemporaines, textes réunis par D. Bouvier, M. Steinrück, Pierre Voelke, Grenoble, Jérôme Millon, 2008. 
du poieîn dans nos propres constructions discursives. Jadis, à l'université de Lausanne, nous avons travaillé en collaboration interdisciplinaire - un mode de travail auquel je tiens beaucoup - sur cette question de la rhétorique du discours des sciences humaines et en particulier du discours anthropologique ${ }^{13}$. Cette approche de la culture grecque antique avec les représentations que l'on tente d'en restituer me conduit à évoquer quelques-unes des étapes de ma formation ; ces étapes me semblent marquer cette image, cette représentation de la Grèce ancienne que je crois avoir donnée sous différents aspects, à travers les ouvrages ou les études que vous avez mentionnés. Cette Grèce, je l'ai d'abord perçue par la langue, et par conséquent dans et par les textes, en raison de ma formation scolaire, puis universitaire.

J'ai suivi le cursus d'un lycée qui était un lycée classique assorti d'un lycée scientifique, le Collège classique cantonal de Lausanne (qui n'était pas encore mixte!) avec du latin en sixième, de l'allemand en cinquième et puis, dès la troisième, un programme de grec qui s'est étendu sur cinq ans avec un enseignement portant naturellement sur la langue, sur la morphologie (surtout la morphologie du verbe qui est particulièrement complexe en grec) et sur la traduction ; avec cette rigueur imposée par le calvinisme diffus qui a toujours marqué et qui marque encore probablement la pédagogie dans les institutions scolaires lémaniques, entre Lausanne et Genève. Puis, à l'université de Lausanne, cette formation s'est poursuivie en somme dans le même sens, avec des exercices de version et de thème d'une part, et d'autre part des séminaires d'explication de textes, essentiellement poétiques. Je me rappelle notamment - j'avais alors à peine dix-huit ans, et cela a certainement orienté le chemin de recherche que j'ai choisi par la suite - une lecture des poèmes fragmentaires d'Alcée et de Sapphô. Le séminaire était animé par un professeur, André Rivier, qui enseignait la langue et la littérature (il était, pour situer un peu le contexte intellectuel qui était le sien, collaborateur pour la Suisse romande de la revue Esprit). Par cet intermédiaire, j’ai été marqué, sans aucun doute, par la philologie allemande, mais surtout celle représentée par les professeurs qui avaient dû fuir le régime nazi pour s'exiler en Angleterre ou aux États-Unis (Rudolf Pfeiffer, Hermann Fränkel); et notre professeur nous a également introduits aux travaux anglosaxons issus de l'École de Cambridge, fortement influencée par l'anthropologie de la fin du XIX ${ }^{\mathrm{e}}$ et du début du XXe siècle (je pense ici à Gilbert Murray, naturellement, et à Jane Harrison). Enfin, à travers les cours donnés par André Rivier, ce sont en particulier les travaux d'Eric Dodds qui m'ont frappé : anthropologie de la distance dans la mesure où elle nous présentait des Grecs irrationnels.

Puis, à André Rivier a succédé François Lasserre, qui m’avait appris à écrire et à lire le grec au lycée - c'était une tradition, à ce moment-là, d'être professeur de lycée avant d'obtenir un poste à l'université. C'est à François Lasserre que j'ai succédé, en 1984, pour cette chaire intitulée "Langue et littérature grecques ». Ce qu'il faut

13 Voir notamment J.-M. Adam, M.-J. Borel, C. Calame, M. Kilani, Le discours anthropologique. Description, narration, savoir, Lausanne, Payot, $1995,2^{\mathrm{e}}$ éd. 
préciser encore - je crois que ce n'est pas tout à fait indifférent -, c'est que nous étions en définitive (je peux dire nous...) les lointains successeurs de Théodore de Bèze, le célèbre théologien protestant, traducteur de la Bible, qui enseigna le grec à l'Académie de Lausanne, au milieu du XVI ${ }^{\mathrm{e}}$ siècle, avant de succéder à Jean Calvin à l'Académie de Genève. Origine de l'Université, l'Académie de Lausanne a été fondée en 1537 après que les Bernois eurent introduit la Réforme dans cette région en 1536. Et pourquoi la fondation de l'Académie une fois la Réforme introduite ? C'est qu'il fallait, tout simplement, former des pasteurs susceptibles de convaincre les ouailles, de les convertir du catholicisme au protestantisme. L'une des deux premières chaires à être créées fut celle de grec ancien. On en revient aux textes. Il s'agissait naturellement de lire le Nouveau Testament en grec, dans cette perspective protestante qui nous ramène au problème de l'explication du texte : qui dit lire le texte, dit aussi le commenter. C'est dire que la lecture dans la langue originale implique l'explication et, par conséquent, une démarche d'ordre herméneutique.

Pour suivre l'itinéraire universitaire qui a été le mien, je dirai qu'une autre étape importante a été le moment où j'ai pu saisir les enjeux des manifestations de la Grèce antique, que je percevais surtout comme textuelles, à partir des préoccupations à la fois idéologiques mais aussi académiques (finalement, l'institution était impliquée) et culturelles contemporaines : en l'occurrence le foisonnement des questionnements et des débats politiques et culturels suscités par le mouvement de Mai 1968, que j’ai vécu d'ailleurs à Hambourg (collaboration scientifique au Lexikon des frühgriechischen Epos, sur la recommandation d'André Rivier), et non pas à Paris, ni d'ailleurs à Lausanne. Si je mentionne cette mouvance colorée de Mai 1968 avec son fort engagement politique, c'est parce que les mouvements étudiants, en particulier, ont donné une impulsion absolument décisive au développement des sciences humaines ; cela pose la question de leur croisement.

Ce que j'ai oublié de dire et ce que je voulais encore préciser, c'est que mon passage dans ce collège classique a été marqué - j’avais alors seize ans - par un voyage en Grèce organisé par François Lasserre. Le tourisme en Méditerranée était à peine développé. Il n'était évidemment pas question de sauter dans un vol d'Easyjet pour rejoindre Athènes en deux heures : train - à vapeur encore ! - à travers toute l'Italie jusqu'à Brindisi ; puis traversée vers Corfou puis à travers le canal de Corinthe pour finalement arriver au Pirée qui correspondait à ce que l'on imaginait d'un port de l'Orient. De plus, le réseau routier en Grèce était encore embryonnaire et la traversée du Péloponnèse était comme un retour au monde pastoral et agraire du XIX ${ }^{e}$ siècle... Donc une expérience de terrain qui, sans doute indépendamment de mon intérêt et de ma sensibilité pour la culture grecque ancienne, me confrontait à une autre réalité contemporaine, une culture paysanne généreuse dans un milieu méditerranéen d'une beauté faite de contrastes, un milieu très différent dans ses couleurs et ses odeurs de la géographie alpine qui m'était familière. Ce fut donc un moment essentiel de ma formation scolaire, dans le cadre d'un lycée tout à fait traditionnel. 
Mais retournons à Mai 68 et au développement des sciences humaines et des sciences du langage. D’abord la linguistique générale, avec une première réflexion suscitée par la lecture des Éléments de linguistique générale d'André Martinet (publié une première fois à Paris chez Armand Colin en 1960) ; puis une orientation vers les problèmes de sémantique et du sens de mots en contexte : lexicographie (Alain Rey et d'autres encore) et sémantique (sémantique structurale, analyse componentielle, etc., notamment La sémantique structurale d'Algirdas J. Greimas, publiée en 1966 à Paris chez Larousse) ; ceci à la faveur de ma collaboration déjà mentionnée au Lexikon des frühgriechischen Epos à l'université de Hambourg, précisément autour de 1968. Donc, linguistique générale, mais je dirais aussi et surtout, anthropologie. À l'évidence, anthropologie culturelle et sociale : en particulier de Bronislaw Malinowski, Une théorie scientifique de la culture et autres essais (Paris, Maspero 1970) et, naturellement de Claude Lévi-Strauss, Anthropologie structurale (Paris, Plon, 1958). Ce sont deux domaines dans lesquels je suis entièrement autodidacte, puisqu'il n'y avait pas de cours de linguistique générale, ni même de linguistique historique à l'université de Lausanne, ni d'ailleurs à Hambourg ; et pas non plus d'anthropologie culturelle et sociale - en tout cas, pas sous cette dénomination-là et pas dans cette acception-là.

Du point de vue de l'approche anthropologique, a été déterminant le double séjour à l'université d'Urbino : dans un premier temps, une année de bourse de recherche offerte (sic!) juste au terme de mes études (cela équivaudrait aujourd'hui à un master, mais c'était, à cette époque-là, simplement une licence), après un mémoire que j'avais consacré à la question de la vérité, de l'alethéia chez Pindare, dans les Épinicies; puis trois ans comme chargé de cours entre 1971 et 1974. Mais pourquoi ce séjour à l'université d'Urbino a-t-il été déterminant ? Il a été marquant pour la perspective anthropologique que vous avez présentée tout à l'heure, notamment celle qui est l'origine de Thésée et l'imaginaire athénien. Je mentionnerai d'abord la collaboration avec Bruno Gentili qui venait d'écrire un article sur le triangle poeta, pubblico e committente. Dans les années 1960, le grand helléniste italien disparu depuis peu insistait sur l'approche compréhensive de manifestations poétiques qui ne peuvent être lues simplement comme des textes, mais qui doivent être replacées dans leur situation d'énonciation, dans leurs circonstances de communication ${ }^{14}$.

Mais alors pourquoi l'anthropologie culturelle et sociale ? C'est que, en ce qui concerne en particulier ces différentes formes de la poésie qu'on appelle la poésie mélique - vous savez que je préfere toujours utiliser la catégorie indigène, pour ne pas utiliser dans ce cas particulier le concept anachronique de "lyrique " - le contexte ethnographique de ces chants à portée rituelle est largement fragmentaire, sinon tout à fait lacunaire. C'est ici qu'intervient l'anthropologie, l'anthropologie sociale des cultures, en combinaison dans ce cas particulier avec l'histoire des religions. L'une et

14 Sur cet aspect des travaux de B. Gentili : Poesia e pubblico nella Grecia antico. Da Omero al V secolo, Roma - Bari, Laterza, 1983. 
l'autre sont fondées sur la comparaison. Partant d'analogies de surface, la démarche comparative ne peut être que différentielle. C'est elle qui nous permet de comprendre les enjeux sociaux et religieux de pratiques et de discours s'inscrivant dans l'ordre de la manifestation culturelle et symbolique. En ce qui concerne la culture antique, nous ne saisissons ces manifestations que par les textes et de manière très fragmentaire.

Mais ce qui a aussi été tout à fait décisif dans mon séjour et dans mon travail de recherche à l'université d'Urbino, c'était le contact avec une culture académique très différente de celle qui avait présidé à ma formation à l'université de Lausanne, marquée par un certain calvinisme. A beaucoup compté aussi l'insertion dans une culture politique et symbolique très différente : celle de l'Italie de la fin des années 1960, où on assistait à l'effervescence politique que vous pouvez vous imaginer, mais aussi, à une expérimentation culturelle extrêmement riche - je me réfere ici, notamment, au domaine du cinéma (Antonioni, Rosi, bien sûr Pasolini et surtout, en ce qui me concerne, Fellini) ; mais aussi à celui de la musique (Luigi Nono) et celui de la littérature (Cesare Pavese, Edoardo Sanguineti, le Gruppo 63, puis Italo Calvino). Donc une espèce de convergence entre ce qui s'est imposé comme une approche anthropologique de la Grèce antique et ma propre confrontation d'ordre anthropologique, d'ordre culturel, avec mon contexte de travail et mon implication politique : contexte académique ouvert aux sciences humaines et, plus généralement, contexte politique et culturel marqué par le marxisme et par des luttes sociales retentissantes.

C'est dans ce milieu-là ou dans ces circonstances-là - il s'agit en fait de circonstances d'énonciation - que j'ai élaboré l'étude sur les deux Parthénées du poète spartiate Alcman. Sur la proposition de Gentili, ce travail doctoral devait correspondre à une édition des fragments d'Alcman avec traduction et commentaire développé. Pour répondre aux critères de la thèse qui à l'université de Lausanne à ce moment-là avait plus ou moins la forme d'une thèse d'État, Rivier, avec raison, a préféré m'orienter vers une étude monographique des Parthénées d'Alcman. En raison de la perspective anthropologique d'emblée adoptée j'ai élargi cette enquête doctorale à toutes les manifestations, en général rituelles, où intervenaient des groupes choraux de jeunes filles. C'est ici qu'est intervenu, à titre comparatif, le concept de " rite de passage " et, puisqu'il s'agissait de groupes d'adolescentes, le concept opératoire de " rite d'initiation tribale ».

Mais - et c'est certainement l'influence de la mouvance de Mai 1968 - s'est aussi imposée la confrontation avec des manifestations d'ordre sexuel et avec d'autres formes de sexualité ; des manifestations fondées sur une conception mais aussi une représentation de pratiques sexuelles évidemment très différentes des nôtres. Je pense aux relations homoérotiques qui sont mises en scène non seulement dans certains poèmes de Sapphô, mais aussi dans les Parthénées d'Alcman, précisément. Manifestations très différentes, dans leurs formes et dans leurs représentations, de la conception très bourgeoise que l'on avait encore dans les années 1960 des relations amoureuses, avec la condamnation morale et sociale que vous pouvez vous imaginer de toute relation d'ordre homosexuel. Dans le cas particulier, les rites d'initiation tribale intégraient des pratiques homoérotiques asymétriques qui permettaient le passage rituel de l'adolescent 
ou de l'adolescente à l'âge adulte, marqué par le mariage " hétérosexuel "; réinterprétation anthropologique de ce qu'on appelle encore à tort "l'homosexualité grecque ". C'est dans cette mesure que je me suis employé ensuite, peut-être pas à montrer, mais au moins à argumenter sur le fait que non seulement notre concept de "sexualité ", mais aussi notre concept d' " homosexualité " ne sont précisément pas pertinents ou à peine pertinents pour les pratiques masculines et féminines et les catégories indigènes concernant l'érôs grec. C'est ici qu'est intervenue, de manière déterminante, la perspective du genre comme ensemble anthropologique des identités et des relations sociales et symboliques de sexe ${ }^{15}$.

Pascal Payen : Et pour ce qui est des traditions savantes dans lesquelles vous vous reconnaissez?

Claude Calame : Sur la question des traditions savantes, des courants d'analyse qui ont permis aux Modernes de tracer une nouvelle carte culturelle de la Grèce ancienne au cours de cette seconde moitié du $\mathrm{Xx}^{\mathrm{e}}$ siècle... J'ai donc mentionné la linguistique générale ; j'ai mentionné l'anthropologie culturelle et sociale. Dans la perspective qui est la mienne, qui dit anthropologie dit naturellement, aussi, histoire des religions ; tout en précisant que, du point de vue de l'anthropologie culturelle et sociale, ce à quoi j'étais plus sensible vers la fin des années 1960, c'était plutôt l'anthropologie fonctionnaliste (j’ai mentionné mes lectures de Malinowski) - une anthropologie centrée sur les pratiques sociales et symboliques, mais une anthropologie également marquée par le marxisme ; avec cette idée qu'en ce qui concerne en particulier la poésie, il faut justement se référer à ce qu'on appelait, dans une approche marxiste ou du moins marxisante, les conditions de production. Nous étions tous, ou du moins une bonne partie d'entre nous, influencés par certaines formes de marxisme.

Ceci pour les pratiques; et les " textes " ? À travers la linguistique générale, la sémantique structurale et puis, du côté de la syntaxe du texte et des formes de discours, la narratologie. Il me semble - je n'en suis pas tout à fait sûr - que c'est paradoxalement par l'intermédiaire de la lecture des travaux de Greimas (que j’ai déjà mentionnés), par l'intermédiaire également des premières esquisses d'analyses narratologiques, que j'ai abordé l'analyse structurale des mythes telle qu'elle a été proposée et illustrée par Lévi-Strauss et telle qu'elle a été pratiquée par Marcel Detienne, en ce qui concerne la Grèce antique. Je rappelle que Les Jardins d'Adonis sont parus dans une première édition en 1972 et que, pourtant voisins très proches des sémioticiens au 10 rue

15 C. Calame, L'Eros dans la Grèce antique, Paris, Belin, 2009 (1 $1^{\text {re }}$ éd. 1996) ; cf. V. Sebillotte Cuchet, «Entretien avec Claude Calame. À la croisée des disciplines », Klio 32, 2010, p. 189-203. 
Monsieur-le-Prince, Vernant et ses collaborateurs éprouvaient une certaine méfiance à l'égard de ce qui se passait juste au-dessus de leurs têtes ${ }^{16}$.

Je crois qu'a été décisive cette combinaison entre d'une part mon intérêt pour la sémantique structurale, et donc pour les valeurs symboliques construites dans le texte (à la fois reproduites et reconstruites dans le texte pour être organisées en représentations discursives), et d'autre part mon intérêt pour la logique d'un texte conçu non pas dans sa fragmentation phrastique, mais dans son ensemble, dans la logique qui le traverse. Croisement donc entre l'intérêt plus proprement linguistique, par l'analyse des discours, et l'approche anthropologique. Avec cette idée que, contrairement à une perspective ou une approche marxisante, il était préférable de partir du texte avec ses marques, avec ses repères énonciatifs et de penser que ces repères étaient indiciels quant au contexte d'énonciation : passer du texte - envisagé désormais comme discours - à son contexte; et non pas reconstruire un contexte pour lire le texte, interpréter le texte lui-même à partir de conditions d'énonciation reconstruites au préalable (ce qui était en somme la démarche que j'avais adoptée dans Les Chours de jeunes filles). Donc, finalement, plutôt une démarche inductive de ce point de vue-là, plutôt qu'une démarche déductive ; une démarche faisant déboucher le repérage des indices d'énonciation sur la pragmatique des discours.

C'est évidemment par ce biais-là que je suis venu, pour l'interprétation des textes envisagés dans leur pragmatique, à une conception de la fiction, surtout de la fiction narrative et poétique, comme un poieîn; un poieîn en tant que fabrication d'ordre culturel avec une fictionnalité, un muthôdes - pour reprendre l'expression qu'on trouve, une première fois, chez Thucydide ${ }^{17}$, mais qui a été reprise ensuite par les orateurs, notamment par Isocrate ${ }^{18}$ - qui correspond à la fiction au sens étymologique du terme : une fiction, certes, mais une fiction qui est une fiction référentielle (si on m'accorde cet oxymore), pour ces récits poétiques que nous appelons les mythes. Et puis, ces dix dernières années, dans le contexte d'une définition nouvelle de l'ethnopoétique, le poème grec, surtout dans ses formes méliques et dramatiques, est désormais envisagé comme accomplissement rituel dans la performance musicale ; dans cette mesure, la poésie grecque est à considérer comme une pratique, une pratique de la voix, une pratique du corps, une pratique ritualisée, ce qui nous reconduit en particulier aux rituels et à l'histoire des religions. De cette manière-là, je crois avoir évoqué les courants en sciences humaines que je pense percevoir, disons, à la source des études que j’ai pu entreprendre.

Corinne Bonnet : Je voulais me greffer sur la question de Pascal avec une sousquestion qui peut être traitée rapidement : avez-vous l'impression, Claude, d'avoir

16 M. Detienne, Les Jardins d'Adonis, Paris, Gallimard, 1972.

17 Thucydide I, 22, 4.

18 Isocrate, Panégyrique, 28. 
restitué, ou travaillé sur une Grèce ? Parce que Pascal a posé la question au singulier : quelle Grèce ? Comment réagissez-vous par rapport à cela ?

Claude Calame : Je suis parfaitement conscient qu'il y a une culture spartiate, qu'il y a une culture athénienne, qu'il y a des cultures de cités - la culture de Lesbos aussi, au VI ${ }^{\mathrm{e}}$ siècle -, des cultures qui sont fondées sur un panthéon spécifique et différencié qui inclut des divinités avec des fonctions singulières et des figures héroïques locales. Chacune de ces cultures civico-religieuses connaît son développement propre, certes en interaction avec ses voisines. Je suis désormais réticent, après avoir été dans un premier temps relativement convaincu, à l'égard des réflexions de Gregory Nagy sur le panhellénisme et l'opposition entre panhellénique et local. Il y a certes cette culture athénienne - Isocrate le prétend lui-même et il a raison - qui devient elle-même, moins au $V^{\mathrm{e}} \mathrm{qu}^{\prime} \mathrm{au} \mathrm{IV}^{\mathrm{e}}$ siècle, une culture plus ou moins panhellénique. Mais je crois qu'avant le IV siècle, il est vraiment difficile de parler de panhellénisme. Sans doute la tradition épique, la tradition homérique telle que nous la connaissons par l'Iliade et l'Odyssée, est-elle dans une certaine mesure une tradition panhellénique. Mais, par ailleurs - on le constate notamment dans certains poèmes de Sapphô - les poètes méliques nous présentent des épisodes de la guerre de Troie dans des versions bien différentes de celles qui sont attestées dans "notre " Iliade et dans " notre " Odyssée ou dans ce que nous avons des Kypria. Ce sont des versions issues de traditions locales; dans chaque cas elles sont adaptées aux circonstances d'énonciation du poème et à la culture civique d'un public assemblé en un espace singulier pour un temps particulier. En anthropologie aussi, on est désormais devenu sensible à l'historicité des cultures de tradition orale. La mondialisation n'a fait qu'accentuer la dynamique du changement, et la distinction entre "sociétés froides " et " sociétés chaudes » n’a plus cours.

Corinne Bonnet : Merci.

Claude Calame : Nicole Loraux l'avait déjà indiqué, il est vain de vouloir parler de "l'homme grec ", mais j'ai aussi des réticences à parler de "la pensée grecque " ou " des Grecs ». D’abord, on devrait dire les Grecs et les Grecques, au moins ! en pensant, par exemple, aux rôles remarquables que jouent les figures féminines dans les tragédies d'Euripide. Alors, parler de la pensée grecque ou de la Grèce, cela me semble difficile autant du point de vue géographique que du point de vue historique. On voit ces différentes « cultures du chant " évoluer en contact et en interaction les unes avec les autres. Il y a tout un réseau interculturel, ne serait-ce que parce que les poètes voyagent ou parce que des poètes comme Pindare de Thèbes composent pour d'autres cités. Je pense que l'image du réseau (qui est à la mode désormais - on sait pour quelles raisons !) serait certainement beaucoup plus appropriée qu'un concept englobant tel que "la Grèce ", « les Grecs » ou « la pensée grecque ». 
Corinne Bonnet : Je pense que c'est le moment de nous tourner vers la salle. Y a-t-il des questions susceptibles de prolonger la réflexion sur les sujets que l'on vient d'aborder?

Iwo Slobodzianek : Vous parliez tout à l'heure de votre intérêt, lors de votre formation, pour la linguistique et les études lexicographiques. Cela me fait penser à un autre chercheur suisse que j'ai beaucoup lu, Jean Rudhardt, et je voulais savoir si c'était quelqu'un qui vous avait marqué dans vos recherches, si ses travaux ont exercé une forme d'influence sur vous. Quelles étaient les formes de collaboration avec lui ? Quelle est la réception de ses travaux selon vous?

Claude Calame : Jean Rudhardt était mon aîné, pas tout à fait d'une génération, mais peut-être d'une vingtaine d'années ou un petit peu moins... Il était professeur à l'université de Genève quand je préparais ma thèse. À la publication des Chours de jeunes filles, j'ai senti, de sa part, certaines réticences ; ses réticences provenaient, me semble-t-il, de l'influence, de la marque du structuralisme sur mes premiers travaux. Rudhardt a toujours maintenu à cet égard une certaine distance, tout en offrant des études qui s'inspiraient finalement de l'analyse structurale. Mais je me suis moi-même fondé sur ses travaux, et notamment sur sa thèse, Notions fondamentales de la pensée religieuse dans la mesure où, me concentrant sur le sens des mots, j’ai été soucieux de restituer les catégories, les représentations et les pratiques "indigènes ". Ce sont des travaux qui m'ont été très utiles ${ }^{19}$. Je pense aussi à l'étude de Rudhardt sur les hymnes orphiques qui a été intégrée à une publication posthume, si je me souviens bien, par les soins de Philippe Borgeaud et de Vinciane Pirenne-Delforge dans un supplément de $\operatorname{Kernos}^{20}$. À plusieurs occasions, j'ai travaillé moi-même, par le biais du papyrus de Derveni, sur les différentes formes de discours orphique, qui sont d'ailleurs à distinguer des « passeports pour l'au-delà » que sont les lamelles funéraires d'or ${ }^{21}$.

19 J. RudHARDT, Notions fondamentales de la pensée religieuse et actes constitutifs du culte dans la Grèce classique. Étude préliminaire pour aider à la compréhension de la piété athénienne du IV siècle, Genève, Droz, 1958 (2éd., Paris, Picard, 1992).

20 J. RudHaRdT, Opera inedita. Essai sur la religion grecque \& Recherches sur les Hymnes orphiques, Ph. Borgeaud et V. Pirenne-Delforge (éd.), Kernos suppl. 19 (2008).

21 C. CALAME, "Figures of sexuality and initiatory transition in the Derveni theogony and its commentary ", in A. Laks et G. W. Most (éd.), Studies on the Derveni Papyrus, Oxford, Clarendon Press, 1997, p. 65-80; "La poésie attribuée à Orphée : qu'est-ce qui est orphique dans les Orphica? Une mise au point introductive ", RHR 219, 4 (2002), p. 385-400 ; "Itinéraires rituels et initiatiques vers l'au-delà : temps, espace et pragmatique dans les lamelles d'or ", in Pratiques poétiques de la mémoire, p. 229-288, "The Derveni papyrus between the power of spoken language and written practice : Pragmatics of initiation in an Orpheus poem and its commentary ", in I. Papadopoulou et L. Muellner (éd.), Poetry as Initiation. The Center for Hellenic Studies Symposium on the 
Il y avait d'ailleurs chez Rudhardt une croyance qui ne rendait peut-être pas la communication extrêmement aisée. Apparemment il éprouvait pour le panthéon grec, en tout cas pour la mythologie grecque, un sentiment et une attitude qui s'approchaient d'une posture de foi. Je vous raconte une anecdote que je trouve tout à fait significative. On m'a rapporté qu'à l'occasion d'un séjour à Athènes, il a eu un malaise cardiaque - vous savez qu'avec la tuberculose, il a été très éprouvé dans sa santé -, il a dû être transporté à l'hôpital, et sa femme, passant sous l'Acropole et voyant le Parthénon, s'est adressée à Athéna pour lui dire, en substance : "Écoute, Athéna, s'il y a encore quelqu'un qui croit en toi ici, c'est mon époux ; je t'en prie, fais quelque chose pour lui, tire-le de cette mauvaise situation. »

Corinne Bonnet : Je souhaite seulement vous poser une question liée à votre livre sur la fondation de Cyrène. Dans le titre, vous avez affiché le terme de "symbolique ». Vous n'êtes pas revenu sur ce choix, et $\mathrm{j}$ 'aimerais savoir où vous situez, dans l'ensemble de concepts, de méthodes que vous avez mis en avant, le registre du symbolique.

Claude Calame : C'est une question complexe. En ce qui me concerne, l'objet de l'anthropologie, ce sont, avant tout, les représentations culturelles, et je pense, de manière très générale, que ces représentations sont de l'ordre du symbolique. De manière plus concrète, je vois le fonctionnement du processus symbolique à l'œuvre, en particulier, dans la création poétique de Pindare, notamment par le jeu des métaphores. C'est ainsi que je tente, dans l'interprétation d'un texte poétique destiné à la performance ritualisée, de dégager les "isotopies ", les lignes sémantiques qui animent le poème et qui organisent sa substance sémantique - souvent par le recours à toute une série de métaphores. Quant à l'étude que vous mentionnez, la représentation narrative de la colonisation de Cyrène s'avère centrée, dans la $4^{\mathrm{e}}$ Pythique, sur le thème de l'autochtonie (par la manipulation réelle de la motte d'un sol productif), dans la $9^{\text {e }}$ Pythique sur le mariage comme métaphore de la productivité agricole et humaine, et dans la $5^{\mathrm{e}}$ Pythique sur le processus d'héroïsation (narratif, puis rituel) auquel contribue la performance du poème lui-même. Je pense qu'on se meut, là, dans l'ordre du symbolique, selon les valeurs et les représentations indigènes; et on pourrait naturellement faire le même discours pour l'iconographie.

Pascal Payen : Je rappelle que le dossier du processus symbolique, tel que vous l'analysez, figure notamment dans Thésée et l'imaginaire athénien, où vous lui consacrez plusieurs dizaines de pages. Désigner votre enquête comme une enquête attentive à un processus culturel associé au registre du "symbolique ", n'est-ce pas le moyen de vous placer à égale distance de l'analyse d'un objet fictionnel, purement fictionnel, et

Derveni Papyrus, Washington DC - Cambridge MA, CHS - Harvard University Press, 2014, p. 165-186. 
de celle d'un objet qui serait, lui, purement historique, "réaliste " ? Entre les deux, à l'intersection des deux, il y a un processus qui se situe à l'écart du fictif, mais également de toute prétention réaliste. Le processus symbolique, en effet, se raccroche à l'un et à l'autre. Du moins est-ce ainsi que j'avais lu ces pages.

Claude Calame : J'allais le préciser. On interroge les textes antiques, on interroge la culture à laquelle on est confronté, qu'elle soit ancienne, moderne ou contemporaine, d'après les préoccupations, notamment conceptuelles ou idéologiques, du moment. À cette interrogation sur le symbolique s'est progressivement substituée l'interrogation sur la fiction, sur le rapport entre le factuel et le fictif, en ce qui concerne notamment le discours. C'est dans cette mesure-là que j'en suis venu, à travers la notion d'un "fictionnel " comme fabrication "poiétique " à partir d'un donné culturel, à l'idée de fiction référentielle (voir supra!). Mais dans Pratiques poétiques de la mémoire, j'ai mis en avant tout ce que la conception grecque classique du poiètès et du poieîn impliquait dans le sens de la création et, finalement, de la création symbolique, même dans le domaine de l'historiographie : création culturelle et donc création symbolique pour engendrer et diffuser des formes de mémoire collective, de mémoire culturelle efficace ; en constant mouvement, cette mémoire culturelle polymorphe est portée par des formes de discours à forte composante pragmatique. En somme, l'objet reste sensiblement le même, mais l'angle d'approche a un peu changé.

Adeline Grand-Clément : On a surtout parlé de textes jusqu'à présent ; je voudrais revenir sur la question des images et de la place des images dans vos travaux, dans le cheminement de votre pensée. Quelles études ont été pour vous stimulantes dans ce domaine et comment avez-vous forgé votre propre méthode d'analyse des images ? De qui vous êtes-vous inspiré ?

Claude Calame : La réponse est simple : ce sont essentiellement les travaux de Claude Bérard qui m’ont influencé. J'avais été très impressionné par son travail de thèse sur les Anodoi et j'ai pu prolonger ma réflexion notamment à travers les contributions réunies dans La Cité des Images ${ }^{22}$. J'ai pu échanger avec lui dès mon retour d'Urbino à Lausanne, et discuter avec lui de ses approches. Par son intermédiaire m’ont aussi marqué les études de l'imagerie grecque proposées par François Lissarrague ou par Françoise Frontisi-Ducroux, sans aucun doute. Tout en précisant que mes lectures d'images se sont toujours tenues un peu à la marge. Elles servent généralement d'arguments pour une démonstration fondée surtout sur les textes, sur les poèmes - je pense, par exemple, à mon travail sur la figure de Thésée et le dithyrambe XVII de

22 Exposition présentée en 1984, avec son remarquable catalogue La cité des images. Religion et société en Grèce antique, Lausanne, Paris, LEP - Nathan, 1984. À ce sujet, voir notamment : F. Frontisi-Ducroux, "L'image et la cité », Mètis 9, 9-10 (1994), p. 199-207. 
Bacchylide, entre " mythe » et « rituel " 23 . Dans d'autres cas, comme dans celui du vase de Pronomos, sur lequel je suis revenu à deux reprises ${ }^{24}$, les images me permettaient de fonder une conception du masque qui ne fait que voiler une identité civique sans permettre d'incarner le personnage qui est joué sur scène : il s'agit d'une mise à distance énonciative, essentielle pour comprendre la portée de l'action hérö̈que dramatisée sur la scène attique. Je m'inscrivais ainsi en faux à l'égard des analyses de Jean-Pierre Vernant ou de Françoise Frontisi, qui adoptent pour le masque grec une conception du masque comme incarnation d'un personnage, traditionnelle en anthropologie culturelle et sociale. Dionysos n'est pas le « dieu-masque »!

Clément Bertau : Je souhaitais aborder avec vous trois questions. La première concernait la catégorie du mythe et sa déconstruction, mais vous êtes revenu assez longuement sur cet aspect. La deuxième porte sur la notion d'interdisciplinarité. Si on fait le constat, aujourd'hui, que l'univers de la recherche parait soumis à une double dynamique d'hyper-spécialisation disciplinaire et de fragmentation des objets d'étude, la démarche interdisciplinaire serait-elle, selon vous, le complément logique de ces phénomènes, dans les sciences humaines comme dans les sciences exactes? Pourrait-elle aussi être envisagée comme le reflet de la complexité des objets qu'on étudie en sciences humaines ? La troisième et dernière question, plus anecdotique mais liée à un moment important de votre parcours, concerne l'enquête anthropologique que vous avez menée durant l'hiver 1981-1982. C'était une enquête de terrain, sur les rives du fleuve Sepik, en Papouasie-Nouvelle-Guinée, parmi les Abelams et les Iatmuls - si j’ai de bonnes informations -, qu' on appelle parfois les " hommes crocodiles", en raison des scarifications qu'ils s'imposent au cours de leurs rites initiatiques. Avec le recul, pensez-vous que cette expérience de terrain a stimulé votre imagination savante, je veux dire en un sens positif, comme aptitude à se représenter, à nourrir d'expériences des concepts théoriques ? Votre souci de la dimension pragmatique des productions symboliques, pour ne mentionner qu'un exemple, a-t-il pu germer au contact d'un objet d'étude plus "vivant", pour ainsi dire?

Claude Calame : Sur la première question que vous avez formulée, celle du mythe et de notre conception des rapports entre mythe et histoire, il y a un point général que j'aimerais encore aborder, parce qu'il m'a passablement préoccupé ces dix dernières

23 C. Calame, "Thésée l'Athénien au Metropolitan Museum of Art de New York : scènes étiologique de légimitation et questions de méthode ", in D. Yatromanolakis (éd.), An Archaelogy of Representations. Ancient Greek Vase-Painting and Contemporary Methodologies, Athens, Institut du Livre - A. Kardamitsa, 2009, p. 98-127 ; voir aussi le chapitre III de Pratiques poétiques de la mémoire.

24 C. Calame, "Aetiological Performance and Consecration in the Sanctuary of Dionysos ", in O. Taplin, R. Wyles (éd.), The Pronomos Vase and its Context, Oxford, Oxford University Press, 2010, p. 65-78, après les chapitres IV et V du Récit en Grèce ancienne. 
années, à travers différents travaux : celui de la traduction ou, plus précisément, de ce que j'ai appelé la "traduction transculturelle ». Avec la Grèce antique - et cela rejoint la première question de Pascal sur l'image que l'on en construit - on a affaire à une culture, à un ensemble de manifestations culturelles qui sont éloignées de nous, à la fois dans le temps, c'est bien évident, mais aussi dans l'espace. Il est important de relever que, du point de vue anthropologique, notre relation avec la culture grecque est à cet égard profondément asymétrique. La relation anthropologique est toujours, d'une certaine façon, asymétrique, même quand elle s'est débarrassée de l'appui colonial : c'est nous, pour nos intérêts propres, qui questionnons les autres. Mais avec les anciens Grecs et Grecques, contrairement à ce qui se passe dans le cas d'un travail de terrain auprès d'une communauté traditionnelle existante, il n'y a pas, à l'évidence, de dialogue possible; il n'y a pas d'échange qui permettrait de réorienter et d'ajuster la perspective, à partir des réponses que l'on reçoit.

De là, me semble-t-il, la nécessité d'autant plus impérieuse des concepts opératoires, dans la mesure où ce sont les opérateurs mêmes d'une traduction transculturelle par définition asymétrique ; de là aussi la nécessité d'être critique vis-à-vis d'instruments conceptuels qui appartiennent à un paradigme académique donné. Sans doute est-ce un peu contradictoire : on a besoin d'instruments conceptuels, de catégories qui sont souvent des catégories semi-figurées, comme opérateurs de traduction ; mais, par ailleurs, on sait que ces instruments sont nos instruments et qu'ils accentuent par conséquent le caractère asymétrique de la relation anthropologique. Dans cette mesure, ils doivent être soumis à la critique. C'est le travail que j'ai tenté de mettre en œuvre pour le concept moderne de mythe, mais aussi, par exemple, pour la catégorie du " lyrique " (auquel je préfere, je l'ai dit, le concept grec de mélos, qui inclut toutes les formes de poésie pratique et ritualisée, souvent chorale, bien éloignées de poèmes lyriques où le poète exprimerait directement ses sentiments les plus intimes). Ce qui est intéressant, c'est que, dans l'opposition entre étique et émique, entre concepts occidentaux ou théoriques et concepts indigènes, on s'aperçoit que, dans les cultures indigènes elles-mêmes, ce qui nous apparaît comme des catégories ou des concepts propres sont l'objet d'une critique. Par l'intermédiaire du muthôdes, les historiens puis les philosophes grecs se sont posé non pas la question de la valeur de vérité historique mais celle de la vraisemblance morale de ces archaia ou palaia que nous englobons dans la catégorie du mythe ${ }^{25}$.

L'autre remarque que je souhaite faire au sujet de ces opérateurs de la traduction transculturelle, qui sont aussi les opérateurs d'une démarche en un certain sens comparative, c'est que, en anthropologie ou en histoire des religions, nous avons tendance à donner à nos propres concepts - celui de "mythe ", autrefois ceux de "tabou ", d' " ordalie ", voire de "rite de passage " ou de "rite d'initiation tribale " - une valeur universelle ; nous tendons à les essentialiser, à les naturaliser, alors qu'ils ne sont 
qu'opératoires et que, dans cette mesure, ils sont, eux-aussi, marqués dans le temps, dans l'espace, liés à une culture académique particulière. Le concept de mythe, dans la mouvance structurale des années 1970 et 1980, n'est certainement pas le même que le concept de mythe pour l'anthropologie critique d'un Clifford Geertz par exemple.

Concernant, ensuite, la démarche transversale - c'était votre deuxième question -, ce que j'essaie de proposer se situe un peu à la croisée d'une analyse des discours (notamment poétiques), attentive aux procédures d'énonciation, et d'une anthropologie culturelle d'ordre forcément historique. Ce croisement s'accompagne aussi, du point de vue disciplinaire, du recours à des disciplines plus techniques, liées aux problèmes posés par les textes : la philologie, entendue comme pratique d'établissement du texte, la dialectologie - vous savez bien que les poèmes de Pindare ou les poèmes des formes méliques offrent des formes qui, du point de vue dialectal, sont différentes du grec parlé dans l'Athènes du v siècle -, la métrique, la papyrologie, la codicologie, etc. Cela pose évidemment le problème pratique de savoir comment croiser ces différentes disciplines, ceci à d'autant plus forte raison que, par exemple, la question très technique du schéma métrique d'un poème de Sapphô a un impact décisif sur la question anthropologique de la performance musicale du poème, avec ses conditions d'énonciation. Le rythme métrique, qui renvoie à une chorégraphie, est une dimension essentielle de la pragmatique du poème.

Cela pose aussi une question institutionnelle, qui me préoccupe beaucoup pour ce qui concerne les travaux de doctorat que j'ai été ou suis encore conduit à diriger. Parce que ce croisement interdisciplinaire, en fin de compte, ne correspond pas aux catégories du CNRS, ni à celles CNU, et je me suis trouvé confronté à des dossiers qui de l'histoire ont été renvoyés vers la littérature, ou de la littérature vers l'histoire ancienne ou l'histoire des religions - vous connaissez bien le problème. Tout ceci pour rappeler que la question épistémologique a aussi un impact d'ordre institutionnel, et je crois que lorsqu'on élabore un projet de recherche avec un candidat en doctorat, il faut songer à cette insertion disciplinaire, même si on ne partage pas ce cloisonnement thématique et épistémologique entre différents domaines.

J'ai également constaté qu'à la suite de l'extraordinaire développement des sciences humaines pendant les années 1970-1980, on a assisté dans les années 1990 à une certaine méfiance ou réticence à l'égard de la théorie, surtout chez les plus jeunes - peut-être le déconstructionnisme, le post-structuralisme ou les pesanteurs de ce que les Anglo-Saxons appellent la " French Theory " ont-il eu une certaine influence à cet égard. Désormais, il est assez difficile d'essayer d'affirmer l'impact positif de la définition d'une approche qui se voudrait transdisciplinaire ou interdisciplinaire. Il y aurait toute une enquête à conduire sur la façon dont cette méfiance vis-à-vis de la réflexion méthodologique, épistémologique, théorique en sciences humaines s'est installée au tournant du siècle nouveau. Les éditeurs vous le disent de manière désormais lancinante : dans le domaine des sciences humaines et en particulier dans celui de l'anthropologie, les tirages sont passés de 2000 ou 3000 exemplaires dans les années 1990 à 
500, voire moins. Ce qui est intervenu ici, c'est l'énorme reflux, je pense, à la fois sur le plan politique et sur le plan moral, de la pensée sociale sous le monopole gagné par l'idéologie néolibérale ; il s'agit de ce libéralisme à l'anglo-saxonne qui imprègne non seulement l'économie, mais aussi la manière de penser ou d'envisager les problèmes sociaux ou culturels sur la base d'un individualisme capitaliste très égoïste - cela depuis, désormais, une bonne vingtaine d'années. Ce fut un vrai tournant idéologique. Et contrairement à ce qu'on aurait pu penser et espérer, la crise financière et économique de 2008 n'y a rien changé, bien au contraire.

Au sujet, maintenant, du travail de terrain et de mon enquête dans le village de Palimbeï en Papouasie-Nouvelle-Guinée. Si j'avais choisi la région du fleuve Sepik, c'était pour deux raisons. Tout d'abord une raison d'ordre anthropologique, car je savais qu'on pratiquait encore dans cette région des rites d'initiation tribale. Ces rites concernaient, en partie, les jeunes filles, sous la forme de rites de puberté, individuels, liés à l'apparition des premières menstrues. Pour les jeunes hommes, les rites d'adolescence étaient encore des rites collectifs ; ils correspondaient donc à notre concept de rites d'initiation tribale. Cette enquête était liée au lancement du projet de recherche sur Thésée et l'imaginaire athénien, envisagé comme une sorte de contrepartie masculine aux Chours de jeunes filles. Je ne pouvais continuer à faire uniquement du travail de cabinet : il s'agissait finalement d'anthropologie comparée, en dépit de la distance historique et de l'éloignement géographique ; il fallait être confronté au terrain.

Viennent ensuite la ou plutôt les raisons pratiques. D'une part, j'étais en contact avec le séminaire d'ethnologie de Bâle qui, régulièrement, accueillait des doctorants qui travaillaient sur deux ou trois villages iatmuls le long du Sepik. Je pouvais donc profiter de leurs travaux pour avoir une certaine connaissance préalable de la communauté et de la culture concernées. C'est d'ailleurs par ce savoir livresque que j'ai appris que les cursus initiatiques n'avaient pas encore disparu des bords du Sepik. D'autre part, raison plus concrète encore, dans cette région on parle - les plus jeunes du moins - le pidgin. Pour une enquête de trois mois, il n'était pas question pour moi d'apprendre le iatmul ; le pidgin m'offrait donc la possibilité de communiquer. Au final, cette expérience de terrain a été déterminante - c'était le sens de votre question - pour donner de la chair aux concepts théoriques; car j'étais déjà bien conscient, avant même ce séjour, que ces concepts théoriques étaient simplement nos instruments de pensée. Ils sont devenus pour moi, plus tard, des instruments de la traduction transculturelle.

Sur le plan pragmatique et pratique - je n'ai évidemment pas pu suivre tout le cursus, mais j'ai pu en observer une ou deux phases -, j'ai éprouvé un étrange sentiment devant ces rites. Je dois préciser, d'abord, que les jeunes hommes en question suivaient par ailleurs un cursus scolaire, introduit par le pouvoir colonial. Il avait été imposé par les missionnaires chrétiens pour être assumé, après l'indépendance, en grande partie par les Papous eux-mêmes. Tout cela a évoqué pour moi le cursus militaire imposé au jeune citoyen helvète. À 19 ans, vous arrivez dans une école de recrues, dans un espace extérieur, aux limites bien définies et à l'organisation propre. Vous arrivez avec 
votre formation de jeune étudiant, avec votre statut civil, avec votre affectivité d'adolescent. Très vite on vous remet solennellement, au-dessus d'une bannière rouge à croix blanche, un fusil d'assaut (que vous emporterez à la maison), et vous acceptez d'endosser le rôle symbolique qui y est attaché, avec ses pratiques fortement codifiées. Vu de l'extérieur, tout cela vous semblait complètement étranger ; mais tout en percevant les enjeux, vous jouez le rôle qui vous est assigné de manière étonnamment sincère. J'aurais voulu faire un travail - je n'en ai malheureusement pas eu l'occasion - sur la ritualité et les rythmes qui scandent la vie militaire, ne serait-ce que par le port de l'uniforme, avec le discours qui l'accompagne et la soutient. Enfin, la vie militaire est faite de conventions imposées, de formes de discours codifiées et de pratiques rythmées qui rappellent ce que nous connaissons par l'anthropologie des pratiques rituelles. L'école de recrues correspond à une formation que l'on reçoit, avec le statut qui vous est attribué au terme de ce cursus de quatre mois, à forte composante initiatique. Qu'il s'agisse de Palimbeï ou de la place d'armes de Bière, pour hasarder une comparaison de surface, m'est apparue l'importance des pratiques collectives et ritualisées pour toute formation à portée " anthropopoiétique " (pour employer un concept opératoire que nous avons forgé en commun ${ }^{26}$ ), quelles qu'en soient l'orientation et la fonction.

Mais pour repasser du pied du Jura aux bords du Sepik, ce qui m’a frappé auprès des Iatmuls puis des Abelams, c'est en définitive une proximité spontanée face aux problèmes pratiques les plus élémentaires. C'est la possibilité d'une communication relativement immédiate, fondée aussi sur le sentiment, la sympathie (ou l'antipathie !), l'attirance affective et sexuelle; c'est aussi la possibilité de l'échange verbal animé par des sentiments communs; et ceci en dépit de différences culturelles et sociales à l'évidence énormes. L'étrangeté se commue en familiarité. Cela m’a beaucoup touché et, en quelque sorte, rassuré. Ce sentiment, je l'ai revécu récemment à Paris même, dans le soutien collectif apporté depuis l'EHESS aux travailleurs sans-papiers en grève, majoritairement africains.

Adeline Grand-Clément : Cette expérience de terrain, dites-vous, a contribué à vos travaux. Avez-vous eu, par la suite, l'occasion d'en faire une nouvelle ? Dans le cas contraire, pourquoi vous en êtes-vous tenu à cela ? Auriez-vous souhaité, pour ce qui vous concerne, renouveler l'expérience?

Claude Calame : Si je n'ai pas renouvelé l'expérience, en tout cas sous cette forme du séjour prolongé, c'est pour des raisons familiales d'une part, contingentes de l'autre. J'aurais souhaité revenir dans la même région du Sepik. En effet, à la suite de mon séjour en PNG, j'ai lu la double enquête d'un anthropologue américain qui est revenu

26 Francis Affergan, Silvana Borutti, Claude Calame, Ugo Fabietti, Mondher Kilani, Francesco Remotti, Figures de l'humain. Les représentations de l'anthropologie, Paris, Editions de l'EHESS, 2003. 
sur le terrain ${ }^{27}$; il a relevé, en particulier, la manière dont sa première intervention sur le terrain avait été intégrée dans les récits ou dans l'histoire de cette communauté. Il remarquait, évidemment, combien la culture avait changé, à ce moment-là, sous l'effet, sinon de la mondialisation, du moins d'une certaine "modernisation ", avec les questions identitaires que cela suppose. Les Papous de son village avaient par exemple supprimé le culte secret des ancêtres qui leur assurait, par l'affirmation de leur masculinité, la domination sur les femmes de la communauté.

Clément Bertau : Je reviens à la question du mythe. Vous avez évoqué cette histoire, cette interprétation du mythe, la science des mythes européenne et la nécessité d'une utilisation critique des catégories opératoires. Peut-on, pour autant, se débarrasser complètement de certains acquis - je pense notamment aux travaux de Bruno Snell et à La Découverte de l'esprit ${ }^{28}$ ? Pour ne citer qu'un exemple, les études philologiques sur l'usage de l'article neutre, sur l'idée d'une plus grande conceptualisation par rapport à la pensée homérique, demeurent-elles encore pertinentes à vos yeux ?

Claude Calame : Avec les Wege und Formen frühgriechischen Denkens d'Hermann Fränkel $^{29}$, les recherches de Bruno Snell m’ont beaucoup apporté. André Rivier nous en avait déjà parlé dans les années 1960, à l'occasion de mon cursus universitaire. J'ai ensuite eu la chance de connaître Snell à Hambourg après l'avoir rencontré à Urbino à l'occasion d'une conférence donnée sur l'invitation de Gentili (la confrontation entre les deux cultures universitaires a pris une tournure pour le moins cocasse). Lors de mon séjour à Hambourg, Snell était à la retraite, mais il tenait encore, de manière tout à fait caractéristique, à donner aux étudiants débutants un proséminaire qui portait alors sur les poèmes élégiaques.

Son Entdeckung des Geistes est un livre paradoxal. D'une part, en effet, Snell essaie de montrer, par exemple dans le premier chapitre qui porte sur la poésie homérique, que la conception du corps et de la personne sous-jacente à l'action de l'Iliade est différente de celle que nous projetons sur le monde grec. Dans sa conception homérique l'action de l'homme serait faite du croisement de différentes forces extérieures qui agissent sur lui, se manifestant par le biais de l'action du menos ou du thumos sur des organes tels les phrenes (le diaphragme ?), la kardia (le cour), etc., dans une anatomie et une psychologie qui ne correspondent pas à nos notions de corps propre et de personne-individu. Je pense que Snell a vraiment ouvert une perspective nouvelle,

27 Donald Tuzin, The Cassowary's Revenge. The Life and Death of Masculinity in a New Guinea Society, Chicago, London, The University of Chicago Press, 1997.

28 B. Snell, Die Entdeckung des Geistes. Studien zur Entstehung des europäischen Denkens bei den Griechen, Hamburg, Claassen \& Goverts, 1955 (3e éd.) ; trad. fr. : La Découverte de l'esprit, Paris, L'Éclat, 1994.

29 H. FräNKEL, Wege und Formen frügriechsischen Denkens, München, Beck, 1960 (2 éd.), et Dichtung und Philosophie des frühen Griechentums, München, Beck, 1962 (2 éd.). 
d'ordre anthropologique, en montrant précisément, sur la base d'une fine analyse lexicale attachée aux valeurs sémantiques propres des termes employés en contexte, que nos concepts ne doivent pas être projetés sur une culture différente. D'autre part - ce qui est surprenant au vu de ce positionnement d'ordre anthropologique - Snell inscrit cette conception homérique de la personne (reprise notamment par Vernant) dans la « découverte de l'esprit » comme s'il s'agissait d'une étape primitive s'inscrivant dans un développement linéaire. La perspective est encore une fois la perspective de l'idéalisme allemand, l'indécrottable perspective hégélienne. C'est ainsi qu'en suivant l'« invention » et le développement de l'esprit, Snell fait succéder à la poésie homérique la poésie qu'il appelle lyrique, en projetant sur elle la conception romantique de la poésie comme expression des sentiments intimes du poète. C'est comme si, par la poésie "lyrique " (en fait mélique), les Grecs avaient découvert l'intimité affective et, par ce biais-là, la notion de personne. De manière non moins surprenante, c'est une idée que Vernant reprend dans sa contribution sur l'individu dans L'individu, la mort, l'amour ${ }^{30}$. En dépit de ce paradoxe fondamental, la lecture des études de Snell (notamment Dichtung und Gesellchaft ${ }^{31}$ ) est stimulante.

Traduit en France en 1969, Le monde d'Ulysse de Moses Finley n'était pas encore connu en Allemagne ${ }^{32}$. Avec la poésie épique grecque, on se situe essentiellement dans l'ordre narratif, dans l'ordre du " récit ". On se meut dans un monde de fiction poétique, quelle que soit la référence historique, d'ailleurs composite, du monde homérique : fiction référentielle! Dans la poésie mélique, au contraire, le récit, le " mythe » est mis en relation énonciative explicite avec le présent, avec le hic et nunc des circonstances d'énonciation. Par exemple, en ce qui concerne certaines compositions de Sapphô ou les Parthénées d'Alcman, les protagonistes du poème en performance disent les sentiments que les choreutes éprouvent ou doivent éprouver dans une situation rituelle donnée, une situation qui à mes yeux est d'ordre initiatique. Au final, les représentations que construisent et véhiculent la poésie homérique, la tragédie ou la poésie mélique sont en fait des représentations liées à une poétique. Cette conscience est encore, me semble-t-il, peu affichée chez Snell, et c'est à l'évidence une dimension qu'il faut introduire de manière forte.

Jean-Claude Carrière : Vous parliez de démarche inductive, en quelque sorte, à partir des textes ou, à la limite, à partir des comportements qu'on observe sur le Sepik. Mais comment échapper aux concepts préconstruits ou préalables, comme celui de

30 J,-P. Vernant, L'individu, la mort, l'amour : soi-même et l'autre en Grèce ancienne, Paris, Gallimard, 1989, p. 211-232.

31 B. SNell, Dichtung und Gesellschaft : Studien zum Einfluss der Dichter auf das soziale Denken und Verhalten im alten Griechenland, Hamburg, Claassen, 1965.

32 M. I. Finley, Le monde d'Ulysse, Paris, Maspero, 1969 (éd. or : 1954 !). 
l'initiation ? Faut-il simplement les faire évoluer ? Ce sont des concepts auxquels on a du mal à échapper quand on aborde un texte.

Claude Calame : J'ai donc dit que ces concepts sont à considérer uniquement comme des notions opératoires, d'ordre instrumental. Ils requièrent un regard critique de notre part, bien qu'ils soient indispensables à la traduction transculturelle. Sur les bords du Sepik, directement confronté à des pratiques que nous appréhendons en termes d'initiation, je me suis dit qu'il fallait aborder le terrain en laissant tout cet appareil conceptuel aux marges de ma mémoire érudite. Mais ces notions sont réapparues dans la réflexion à laquelle a pu me porter, tout simplement, ce que j’ai vu, et ce dont j'ai pu discuter avec quelques-uns des protagonistes de ces rites. C'est la raison pour laquelle j'affirme que la comparaison anthropologique, non seulement est asymétrique, mais, de fait, européocentrée. Il ne faut pas se faire la moindre illusion, je crois, à ce propos. Dans la confrontation de terrain, les autres portent aussi un certain regard sur notre culture ; ils font assurément preuve d'une certaine curiosité à l'égard de ce que nous représentons. On m'a régulièrement demandé, quand on me racontait une histoire - c'est-à-dire, pour nous, un mythe -, de narrer, à mon tour. En général, je leur racontais d'ailleurs des mythes grecs, mais Guillaume Tell fut aussi de la partie.

Pascal Payen : Ce qui renforce l'affirmation que vous venez de formuler, en disant que notre position est forcément européocentrée.

Claude Calame : Oui, nécessairement européocentrée, et ethnocentrée. Ce qui n'empêche pas de faire en effet évoluer les notions instrumentales, indispensables opérateurs de la traduction transculturelle. C'est ce que j'ai tenté de faire en situant le concept d'initiation (tribale) dans la catégorie plus englobante et explicite de l'anthropopoiésis ; je l'ai fait dans le collectif déjà mentionné.

Jean-Claude Carrière : À partir du moment où un Iatmul ou un aborigène essaie d'étudier sa propre culture, de quels concepts dispose-t-il autres que de sciences préconstruites, extérieures à sa propre culture ?

Claude Calame : Certes, je n'ai pas procédé à une véritable anthropologie de terrain, mais dans ce que j'ai lu - c'est vrai aussi, du reste, pour les anthropologues qui travaillent sur des phénomènes culturels contemporains, dans nos propres sociétés -, le moment de la confrontation, de la discussion et de la restitution du résultat de l'enquête aux personnes qui en ont été l'objet suscite des réactions très diverses. Parfois, cela se passe très mal, parce que les personnes n'acceptent pas forcément l'image qui est donnée d'elles-mêmes; parfois, au contraire, cela renforce certaines de leurs propres représentations, certaines de leurs propres affirmations identitaires. Cela dépend de la manière dont l'enquête est conduite, du type de discours choisi et de ce qui est dit par 
l'anthropologue sur les personnes qui ont été ses partenaires sur le terrain. J'ai l'impression qu'il n'y a pas de règle de ce point de vue-là.

Jean-Claude Carrière : Cela ne les fait pas rire, à certains moments, de voir notre explication, notre compréhension des phénomènes observés?

Claude Calame : Ah, sans doute! C'est ce qui m'a beaucoup frappé en PapouasieNouvelle Guinée. Le Sepik est toujours en crue en hiver (l'hiver pour nous...) ; lors de mon séjour, cependant, la crue s'est manifestée de manière prématurée. Pour ne pas être bloqué, j'ai dû quitter Palimbeï plus tôt que je ne le pensais et je me suis déplacé à la fin de mon séjour dans les montagnes, près de Mount Hagen, une petite bourgade dans laquelle il y avait un cinéma. Au cours de ces soirées tropicales où l'on s'ennuie à mourir, je suis donc allé au cinéma. J'étais évidemment le seul blanc et on passait un film comique américain : Airplane ( $Y$ a-t-il un pilote dans l'avion ?). J'ai très vite remarqué que le public riait à des occasions autres que les scènes qui, moi-même, me faisaient rire. Donc, pour toute une série de raisons, la perception et la réception du film étaient différentes.

Corinne Bonnet : Je voudrais parler du comparatisme, qui occupe une place importante dans votre œuvre. Cette question, me semble-t-il, suit un cheminement analogue à celui qui traverse toute votre carrière, toute votre œuvre. Tout d'abord, on rencontre un jeune chercheur passé par la Suisse, Hambourg, Paris, Londres, l'Italie. Ce sont donc une pluralité de traditions scientifiques qui ont fécondé votre pensée. On a ainsi un peu l'impression en vous lisant que le comparatisme vient naturellement, puisque vous êtes habitué à naviguer entre plusieurs façons de faire. Dès votre travail sur Les Choeurs de jeunes filles, en 1977, vous consacrez plusieurs pages à la méthode comparative dans l'analyse du rite. Dès le début, donc, la méthode comparative est présente, une méthode comparative qui trouve chez Lévi-Strauss son point de référence. À cette époque-là, vous suggérez que " méthodologiquement, l'analyse structurale, telle qu'elle a été définie par Lévi-Strauss, constitue l'un des moyens les plus sûrs pour permettre une comparaison rigoureuse ${ }^{33}$ ", en vous opposant - notamment dans un long compte rendu ${ }^{34}$ publié 1971 , avant votre thèse - à la méthode comparative mise en place par Angelo Brelich, auteur du volume Paides e Parthenoi ${ }^{35}$. Angelo Brelich est alors un des grands maîtres de l'école «storico-religiosa " de Rome ; il avait inclus les rites d'initiation tribale dans son étude des rites d'initiation des paides et des parthenoi en Grèce. Vous décrivez alors l'approche de Brelich comme une approche de

33 C. Calame, Les Chours de jeunes filles, p. 32.

34 C. Calame, "Philologie et anthropologie structurale : à propos d'un livre récent d'Angelo Brelich ", Quaderni Urbinati di Cultura Classica 11 (1971), p. 7-47.

35 A. Brelich, Paides e Parthenoi, Rome, Ateneo, 1969. 
type historique, tandis que vous privilégiez une approche définie comme structurale. On retrouve ensuite l'articulation entre initiation grecque et initiation tribale, par le biais de la comparaison, dans votre Thésée, avec comme point d'approche particulier l'articulation entre mythe et rite, et le souci de travailler à la fois sur les analogies et les différences. Il est en effet essentiel de marquer les différences et, il faut y insister - car c'est un apport marquant de votre travail de comparatiste -, de comparer sans essentialiser, en évitant de naturaliser les concepts, notamment ceux de " mythe » et de « rite ».

Or, vous y êtes revenu à plusieurs reprises, notamment dans un bel article de Kernos, " "Mythe" et "rite" en Grèce : des catégories indigènes ${ }^{36}$ ? ", la comparaison est un outil qui protège contre le risque de projeter nos catégories modernes sur les catégories indigènes, qui permet de résister à la tentation d'établir des correspondances entre les nomenclatures indigènes (en l'occurrence grecques) et nos propres nomenclatures. Dans le volume que vous avez publié en 2006 avec Maya Burger intitulé Comparer les comparatismes $^{37}$, émerge en outre l'idée importante qu'il y a plusieurs façons de comparer, plusieurs comparatismes, une conviction que vous illustrez à nouveau dans le volume paru à Liège en 2012, avec Bruce Lincoln, sous le titre Comparer en histoire des religions antiques ${ }^{38}$. Dans l'introduction, vous montrez bien qu'on compare, au fond, depuis Hérodote, et même probablement, dès avant Hérodote, qu'on compare chez les linguistes qui s'intéressent au monde indo-européen, dans Le Rameau d'or de James Frazer, ou encore parmi les historiens des religions du $\mathrm{Xx}^{\mathrm{e}}$ siècle : Marcel Detienne et Jean-Pierre Vernant, Georges Dumézil et Claude Lévi-Strauss. Bref, dans cette grande variété de pratiques, tantôt diachroniques, tantôt synchroniques, tantôt génétiques, tantôt typologiques, ou encore fonctionnelles, le comparatisme est fondamentalement expérimental et exploratoire. Il importe donc de le défendre, voire de le réhabiliter à une époque où le repli disciplinaire menace. Dans cette optique, vous avez invité à repenser les opérations comparatives à travers la figure du triangle comparatif. Pourriezvous revenir sur cette proposition et nous dire ce qui, à vos yeux, reste aujourd'hui du comparatisme de Lévi-Strauss qui semble avoir été votre point de départ, puisque vous affirmiez que " le comparatisme c'est la structure »?

Claude Calame : D'abord, merci pour ce parcours : j'en reconnais volontiers les étapes. J'avais pratiquement oublié la recension de Paides e Parthenoi parue dans les Quaderni Urbinati. J'ai connu Angelo Brelich à Rome même, à l'occasion de mon séjour à Urbino, et c'est là que j'ai appris - j’avais déjà engagé mon travail de thèse

36 C. CAlame, " "Mythe" et "rite" en Grèce : des catégories indigènes ? ", Kernos 4 (1991), p. $179-204$.

37 C. Calame, M. Burger (éd.), Comparer les comparatismes. Perspectives sur l'histoire et les sciences des religions, Paris, Milan, Edidit-Arché, 2006.

38 C. Calame, B. Lincoln (éd.), Comparer en histoire des religions antiques. Controverses et propositions, Liège, Presses universitaires de Liège, 2012. 
sur les chœurs de jeunes filles et je voulais envisager ces pratiques chorales et rituelles dans la perspective des rites d'initiation tribale - que Brelich travaillait sur le même sujet pour les jeunes gens et les adolescentes. Je l'ai donc rencontré et il m'a passé une partie de son manuscrit, ce qui m'a permis de réorienter un peu mon travail. Paides e Parthenoi a donc été publié et j'en ai fait une recension dans les Quaderni Urbinati : j'y oppose la perspective que j'allais exposer dans mon livre, fondée sur un comparatisme anthropologique d'inspiration structuraliste, à la perspective historico-religieuse, «storico-religiosa » comme vous l'avez dit, qui était celle de Brelich. Il a d'ailleurs - je l'ai su indirectement - très mal pris ce compte rendu, parce qu'il était farouchement opposé aux perspectives synchroniques proposées par l'anthropologie structurale. Pour moi, dans cette première phase de ma réflexion et de mon travail de recherche, en me fondant sur les principes affirmés par Lévi-Strauss, il s'agissait d'adopter une démarche précisément en synchronie, indépendamment de toute considération d'ordre historique, et non pas en diachronie. C'est l'un des principes qui fondent l'analyse structurale, de même que les entreprises comparatives et les recherches sur les mythes conduites par Marcel Detienne, surtout dans Les Jardins d'Adonis, où ni la dimension historique, ni les formes des discours retenus ne sont prises en compte.

Dans Les Chours de jeunes filles, en ce qui concerne l'analyse des mythes, j'ai moi-même superposé différentes versions sans prendre en compte ni leurs conditions d'énonciation ni leur historicité. Avec Thésée - cela représente d'ailleurs un des paradoxes du livre, ou même un déséquilibre -, j’ai adopté une perspective de narratologie structurale, travaillant pratiquement en synchronie à partir de la Vie de Thésée de Plutarque (qui allègue d'ailleurs plusieurs versions de certains épisodes) ; puis pour les cultes en relation étiologique avec ces épisodes, j'ai agi en historien des religions imbu d'anthropologie structurale. Mais, comme il s'agissait de la cité d'Athènes et de son histoire avec le développement de la légende de Thésée et des rites correspondants dans le courant des $\mathrm{VI}^{\mathrm{e}}$ et $\mathrm{V}^{\mathrm{e}}$ siècles, je me suis rendu compte qu'on ne pouvait pas mettre la dimension historique entre parenthèses. De là est né le dernier chapitre, mais c'est pratiquement celui avec lequel j'aurais dû commencer : un chapitre de mise en perspective comparative, dans la diachronie, des différentes versions, y compris les plus anciennes, des épisodes de la vie de Thésée que j'avais pris en considération, en relation avec le contexte politique et culturel évolutif de l'Athènes classique.

Bien plus tard, avec les recherches plus théoriques publiées successivement en compagnie de Maya Burger, puis de Bruce Lincoln, il s'est agi de montrer qu'il y a plusieurs manières de comparer, que chacune de ces démarches comparatives peut avoir sa productivité, dans la mesure où la comparaison correspond à une démarche différentielle ; c'est un principe qui devrait s'appliquer à toute démarche comparative. Cela signifie que, dans un premier temps, on s'appuie sur des analogies de surface qu'il s'agit évidemment de ne pas naturaliser ou universaliser ; mais à partir des analogies la démarche comparative conduit à identifier les spécificités dans la confrontation et par contraste. C'est pourquoi la perspective historique est aussi importante : c'est celle qu'a défendue par exemple l'islamologue Lucette Valensi dans sa réponse à Marcel 
Detienne dans un numéro des Annales consacré à la démarche comparatiste ${ }^{39}$, suite à une journée organisée autour de Comparer l'incomparable ${ }^{40}$. Je suis en revanche moins convaincu de la nécessité de prendre nécessairement en compte les contiguïtés géographiques.

C'est par ce biais que j'en suis venu à l'idée du triangle comparatif (que l'on peut développer en polygone). En travaillant sur la notion de mythe ou celle de rite d'initiation tribale, il m'est apparu que, dans toute démarche comparative, les protagonistes sont au moins trois : le comparandum, le comparatum et le sujet, le comparans, c'est-àdire celui qui compare. Cette réflexion m'a conduit à m'interroger sur le regard que nous portons, à travers la démarche comparative, au sommet du triangle ; c'est, par comparaison avec une autre culture, la démarche qui induit un regard oblique, décentré, vis-à-vis de la culture qui nous intéresse, en l'occurrence celle de la Grèce ancienne. Ce même regard nous engage à revenir de manière critique sur nos propres pratiques et sur notre propre culture. Il nous invite à porter un regard oblique et décentré sur nos propres pratiques académiques et nos propres catégories ; en ce qui me concerne, sur le tabou, le rite, le mythe, par exemple, mais aussi, de manière plus générale, sur le paradigme à la fois social, économique, politique et culturel dans lequel nous vivons. En somme, le regard comparatif est devenu une sorte de geste réflexif ou auto-réflexif, mais aussi un regard qui nous engage à l'action politique, au sens grec du terme, hic et nunc. Sans doute est-ce aussi ce regard critique qui m'a toujours tenu dans une position marginale vis-à-vis des institutions et à l'égard des disciplines.

Corinne Bonnet : Pour prolonger un instant encore cet échange autour du comparatisme, je voudrais revenir sur Jean-Pierre Vernant. 2014 est l'année du centenaire de sa naissance. Nous organisons à Toulouse deux rencontres scientifiques autour du concept de "puissance divine ", dans une dimension évidemment comparative. Or, dans le fascicule 7 d'Anabases, en 2008, nous avons publié un inédit de Vernant ${ }^{41}$, une interview qui était restée inédite, où il disait, à propos de ses relations avec Dumézil et Lévi-Strauss : "J'avais des inquiétudes sur la possibilité d'un comparatisme généralisé ${ }^{42}$. »Il avouait même avoir ressenti dans l'approche de Lévi-Strauss une menace d' "a-historisme ${ }^{43}$ ". Pour sa part, ajoutait-il, il préférait voir comment les choses bougent, tout en se situant à l'intérieur du système grec, non sans s'ouvrir à la comparaison, comme dans sa leçon inaugurale au Collège de France en 1975, intitulée

39 L. VALENSI, "L'exercice de la comparaison, au plus proche, à distance : le cas des sociétés plurielles ", Annales HSS 57, 1 (2002), p. 27-30. M. Detienne n'a pas désiré publier sa propre contribution à cette journée.

40 M. Detienne, Comparer l'incomparable, Paris, Le Seuil, 2009 (1 ${ }^{\text {re }}$ éd. : 2000).

41 J.-P. Vernant, "Comme une barque sur un fleuve..." ", entretien inédit avec J. O. Guimarães, Anabases 7 (2008), p. 17-32.

42 Ibid., p. 25.

43 Ibid., p. 26. 
Religion grecque, religions antiques ${ }^{44}$. Quelles affinités ressentez-vous avec la personne et l'œuvre de Jean-Pierre Vernant ? À le lire et à vous lire, on sent bien que vous ne pratiquez pas le même comparatisme, que votre approche du monde grec se déploie différemment. Dans le volume récemment publié à Liège, vous reprenez ainsi l'analyse du mythe des races de Vernant, en commençant par dire que ce n'est de fait ni d'un mythe, ni de race qu'il s'agit. Ce qui est une façon de prendre une distance assez considérable par rapport à Vernant.

Claude Calame : Je l'assume ! Je noterai, en me mettant moi-même en marge, ce que Detienne a souligné et reproché à Vernant, encore de son vivant : c'est que, par rapport à l'idée fondatrice du Centre Gernet, par rapport aussi aux premières enquêtes comparatives conduites par Vernant en collaboration avec divers collègues, notamment les Problèmes de la guerre en Grèce ancienne ${ }^{45}$, on perçoit une espèce de renfermement dans la culture grecque. Mais autant pour la démarche comparative que pour l'analyse structurale, à Vernant il faut associer Detienne, dont l'influence sur mes travaux a été importante, surtout à l'époque du développement de l'analyse structurale des mythes. Mais, à la réflexion, je crois que dans mes lectures comme dans mes contacts, je me suis peut-être senti dans ces années-là plus proche de Pierre Vidal-Naquet. C'est peut-être aussi une question d'attitude militante, mais pas uniquement.

Ce qui m'a toujours frappé chez Vernant, c'est que, contrairement d'ailleurs à ce qu'affirme Sarah Johnston dans un dernier numéro d'Asdiwal ${ }^{46}$, il était certes tout à fait remarquable dans la confrontation des textes, dans la manière de les faire jouer les uns avec les autres, mais pas dans leur lecture attentive. Je trouve qu'il est régulièrement entraîné par une rhétorique absolument extraordinaire, une rhétorique orale qu'il parvient à reproduire à l'écrit. Elle évoque pour moi le charme envoûtant que Gorgias prêtait au discours bien tourné. Il y a d'ailleurs, dans la manière de Vernant d'écrire et de donner ses cours, des tournures qui rappellent celles que Gorgias décrit, par exemple, dans l'Éloge d'Hélène. Cette manière de dire (et d'écrire) renvoie en fait à une forme de rationalité qui a trouvé dans le structuralisme - mais pas uniquement en lui - une forme de réalisation. Je pense surtout à des études comme celles sur le sacrifice, sur le mythe de Pandora, ou celle, un peu plus ancienne, sur le " mythe des races "; j'ai mentionné aussi l'étude sur l'apparition de l'individu et la question de la personne dans la Grèce archaïque et classique. En dépit d'une perspective anthropologique qui est très stimulante, Vernant se laisse souvent entraîner par sa rationalité, au détriment de la logique et de la sémantique des textes (sans compter leur pragmatique). Je trouve, en fait, que le meilleur Vernant, celui que je trouve absolument insurpassable, c'est

44 J.-P. Vernant, Religion grecque, religions antiques, Paris, Maspero, 1976.

45 J.-P. Vernant (éd.), Problèmes de la guerre en Grèce ancienne, Paris, La Haye, Mouton, 1968.

46 Entretien avec S. I. Johnston et F. Graf, Asdiwal7 (2012), p. 21-40. 
celui de la collaboration avec Detienne, dans le livre auquel je me réfere toujours et encore régulièrement - le livre sur l'intelligence rusée ${ }^{47}$. La perspective de collection et d'identification des jeux métaphoriques entre les données et représentations de culture qui est celle de Detienne et celle d'organisation rationnelle et d'intelligence anthropologique qui est celle de Vernant se complètent parfaitement ; c'est aussi fructueux que satisfaisant.

Iwo Slobodzianek : Vous souligniez tout à l'heure, à plusieurs reprises, la nécessité de disposer de concepts opératoires et d'adopter en même temps une attitude critique vis-à-vis d'eux. La plupart de vos travaux sur le comparatisme sont collectifs. Est-ce à dire que la mise à distance des concepts implique nécessairement un travail collaboratif, une multiplicité de regards?

Claude Calame : En réfléchissant sur les démarches comparatives de manière générale, je n’ai jamais su comment résoudre la question que pose notamment Detienne, qui est en fait la question du comparans : doit-il être unique, faisant jouer deux cultures autres, ou, au contraire, polyphonique, impliquant non pas deux mais plusieurs cultures ? C'est un véritable problème. La première solution a été adoptée par Geoffrey Lloyd, dans la mesure où, helléniste de formation, il a appris le mandarin et qu'il le lit couramment. Fondamentalement, le problème qui se pose est celui de la lecture des textes, et par cet intermédiaire, de la connaissance intime d'une autre culture historique - de manière à aborder de manière symétrique comparatum ou comparandum. Mais, même chez Lloyd, je ne suis pas toujours convaincu que la comparaison soit absolument équilibrée. La seconde formule correspond, en ce qui concerne la Grèce ancienne, à la recherche comparative à plusieurs, telles celles qu'a animées Detienne lui-même sur les tracés de fondation ou les modes de l'assemblée. C'est lui, dans l'introduction aux ouvrages collectifs correspondants, qui tente de tirer quelques fils comparatifs, souvent de manière fort intéressante et habile, mais ses collaborateurs quant à eux limitent leur enquête à la culture dont ils sont les spécialistes. Au lecteur de faire le travail de comparaison différentielle.

Corinne Bonnet : C'est une juxtaposition en fait.

Claude Calame : Oui, c'est une juxtaposition. Les collaborateurs se meuvent dans leur propre domaine et se gardent bien de toucher à la culture grecque ou à la culture du voisin. C'est un peu dommage, mais on ne peut pas en faire le reproche à Detienne. Il y a aussi les grandes entreprises comparatives, comme celles de Dumézil, mais qui

47 J.-P. Vernant, M. Detienne, Les Ruses de lintelligence. La mètis des Grecs, Paris, Flammarion, 1989. 
suscitent les objections que nous leur avons adressées dans l'introduction au collectif publié avec Bruce Lincoln.

Marylène Lebrère : J'aurais aimé savoir, comme vous vous êtes confronté au travail de terrain, ce qui a retenu votre attention dans le travail de Philippe Descola, notamment dans son ouvrage, Par-delà nature et culture ${ }^{48}$, où il propose divers outils conceptuels et des ontologies, notamment l'analogisme.

Claude Calame : C'est un livre que j'apprécie, notamment parce qu'il revient, de manière critique et pertinente, sur le partage structural entre " nature " et " culture " qui est finalement une création européenne, marquée dans le temps. Mais j'hésite beaucoup dans la définition de ces quatre paradigmes qui correspondraient à quatre modes de pensée, quatre " ontologies " : animisme, totémisme, analogisme et naturalisme. On assiste à une sorte de retour à l'idée de "pensée mythique " comme stade du développement de la pensée humaine, qui s'avère finalement être celui de la pensée européenne ou occidentale. C'est ce qui me gêne aussi à la lecture du Vernant des Origines de la pensée grecque ${ }^{49}$, un livre encore très marqué par le passage du muthos au logos. Lisez une édition plus récente et vous y trouverez une postface dans laquelle il revient sur cette question ; malgré tout, le logos reste cette forme de rationalité qui est aussi la sienne. Elle préside à son écriture ; elle est présente en filigrane dans sa pensée, peut-être en raison de sa formation de philosophe. Je crains donc qu'avec les quatre catégories définies par Descola, on en revienne à dessiner un parcours du même type, ou alors à faire une histoire des mentalités. Cela dit, c'est un livre avec lequel je dois encore travailler avant de me prononcer. Dans une contribution en cours d'élaboration sur l'opposition "nature vs culture " et à la suite de mon travail critique sur Prométhée généticien ${ }^{50}$, j'aimerais ajouter un cinquième paradigme, le nôtre : le "biologisme " qui permet de dépasser l'opposition "nature-culture " tout en nous mettant en garde à l'égard des causes et des conséquences du changement climatique, par exemple. Mais c'est là une autre histoire qui met en cause le mode de production capitaliste et l'idéologie néo-libérale qui le soutient.

Corinne Bonnet : Je souhaiterais clore par une petite lecture, en relation avec le cheminement, la métaphore de la marche.

Claude Calame : Elle m'est chère! Et elle est fondée sur une pratique polymorphe qui m'est vitale.

48 Ph. Descola, Par-delà nature et culture, Paris, Gallimard, 2005.

49 J.-P. Vernant, Les Origines de la pensée grecque, Paris, PUF, 2007 (1 ${ }^{\text {re }}$ éd. : 1962).

50 C. Calame, Prométhée généticien. Profits techniques et usages de métaphores, Paris, Encre marine - Les Belles Lettres, 2010. 
Corinne Bonnet : On a vu que dans une vie et dans une ouvre on marche et on évolue. Et il y a dans les Sentiers transversaux une note de l'auteur dont j'aimerais lire un passage que je trouve vraiment très beau :

"Sans doute la dédicace appartient-elle au genre de l'essai, monographie ou recueil d'études, en apposant aux lignes qui suivent une sphragis globale, je tiens à exprimer une pensée d'affection toute particulière pour les Rochers de Naye dont les pentes, dès mon enfance, année après année, saison après saison, ont tour à tour accueilli souliers de marche, peaux de phoque, chaussons d'escalade ; du sommet de la Dent de Jaman, lieu inattendu d'un acte nourricier montagnard, au Col de Sautodo associé aux cendres d'un être tout proche, tous deux suspendus entre les constructions de rochers gris striés de couloirs verdoyants et la densité bleue de la surface vaporeuse du lac. Des naissances aux décès, la scansion temporelle des étapes auxquelles confronte la vie partagée avec ses proches se combine subtilement avec l'agencement spatial des volumes mouvants d'un paysage préalpin : épouse, fils, mère, père, il y a longtemps, frère, grands-parents jadis, ils sont présents en filigrane, à différents titres, mais toujours associés à la marche. Car c'est en particulier le rythme de la marche, sous ses différentes formes, qui permet de percevoir et de construire à chaque fois un paysage dans son relief, d'en concevoir l'architecture dans ses formes et ses couleurs, d'en habiter l'atmosphère dans la sensation des densités lumineuses, de conférer à la cadence de l'existence une consistance spatiale.

Rythme profondément organique d'ascensions qui, dans l'effort physique et respiratoire, configurent peu à peu les surfaces des pâturages en devers et les volumes des masses rocheuses, avant le plongeon, par la Piste du diable, dans la brume bleutée qui atténue le contour du lac. Itinéraires organiquement rythmés, itinéraires colorés au grès des saisons par le blanc moiré des narcisses, le rose-rouge irisé des rhododendrons ou l'éclat bleuté des gentianes, itinéraires d'une élégance discrète, collant aux formes d'un relief ; des parcours montagnards qui se sont révélés un stimulant constant de la réflexion critique, mais aussi un moyen thérapeutique efficace à l'égard des anxiétés déstabilisantes qu'entretient un travail universitaire qui se coupe trop souvent de la réalité des êtres et des choses. Le rythme de la marche alpine comme scansion de l'existence avec ses aléas et comme psychotrope ${ }^{51} \ldots$ ”

Pour terminer nos échanges, il me semblait enrichissant d'évoquer brièvement l'imaginaire de Claude Calame. Merci à vous tous, merci surtout à Claude de s'être soumis à cet exercice exigeant.

Claude Calame : Mais stimulant !

51 C. Calame, "Note de l'auteur ", in Sentiers transversaux, p. 11. 